\title{
Mathematics difficulties in extremely preterm children: evidence of a specific deficit in basic mathematics processing
}

\author{
Victoria Simms ${ }^{1}$, Camilla Gilmore², Lucy Cragg ${ }^{3}$, Neil Marlow ${ }^{4}$, Dieter Wolke ${ }^{5}$ and Samantha Johnson ${ }^{1}$
}

\begin{abstract}
BACKGROUND: Extremely preterm (EP, <26wk gestation) children have been observed to have poor academic achievement in comparison to their term-born peers, especially in mathematics. This study investigated potential underlying causes of this difficulty.
\end{abstract}

METHODS: A total of 219 EP participants were compared with 153 term-born control children at 11 y of age. All children were assessed by a psychologist on a battery of standardized cognitive tests and a number estimation test assessing children's numerical representations.

RESULTS: EP children underperformed in all tests in comparison with the term controls (the majority of $P_{s}<0.001$ ). Different underlying relationships between performance on the number estimation test and mathematical achievement were found in EP as compared with control children. That is, even after controlling for cognitive ability, a relationship between number representations and mathematical performance persisted for EP children only (EP: $r=0.346, n=186, P<0.001$; control: $r=0.095, n=146, P=0.256$ ).

CONCLUSION: Interventions for EP children may target improving children's numerical representations in order to subsequently remediate their mathematical skills.

D espite sustained increases in survival rates for children born extremely preterm (EP; <26 wk gestation) (1), the prevalence of severe neurodevelopmental impairments has remained relatively static, and cognitive deficits continue to be the most prevalent disability (2). In addition to global cognitive impairments present in up to $45 \%$ of survivors (3), specific neuropsychological difficulties include deficits in attention (4), executive function $(5,6)$, working memory (7), processing speed (8), and visuospatial skills (9). These are evident even in the preschool years $(10,11)$ and contribute to the poor educational outcomes observed in this population $(3,12)$. EP children also have specific difficulties in processing simultaneously, rather than sequentially, presented information (13).

Although there is considerable individual variation in outcomes, as a group, EP children have poorer academic attainment than term-born peers across all school subjects $(9,14)$ and up to two-thirds have some special educational needs (3). One of the most consistent findings is that EP children have specific difficulties with mathematics that impact markedly their academic attainment $(13,15)$. When comparing EP children with term-born peers, the most substantial deficits are consistently in mathematics. In contrast to reading performance, group differences in mathematics performance remain after controlling for neurosensory impairments or general cognitive ability $(3,16)$.

As yet, little is known about the specific nature of mathematics difficulties in preterm populations, and there is a paucity of studies investigating the underlying mechanisms that may account for these deficits (17). Emerging research with typically developing children has revealed that both domain-general and domain-specific skills play critical roles in individual differences in mathematical attainment (18). Domain-general skills observed to be important predictors of attainment in mathematics include visuospatial skills, working memory, shifting, and inhibitory control; $(19,20)$ there is also evidence that language abilities perform an essential role (21). In addition, domain-specific skills such as retrieval speed of answers (22), use of efficient strategies, (23) and procedural competency (24) also contribute to mathematical success. In particular, the accuracy and precision of internal numerical representations, typically assessed using measures of children's estimation skills or the ability to enumerate or discriminate between quantities, have been found to be predictive of achievement in mathematics (25).

Investigating EP children's numerical representations and mathematical processing in detail is thus an important first step in understanding their mathematical difficulties and in developing targeted interventions for this group. This study aimed to investigate the association between numerical representations and attainment in mathematics and to identify domaingeneral and domain-specific processes that may underlie poor mathematical attainment in EP children.

\section{RESULTS}

Effect of EP Birth on Standardized and Experimental Tests

Control children completed all the tasks, except for one child who did not complete the Mathematics Estimation Test (MET) due to time constraints. Three EP children were unable to

\footnotetext{
'Department of Health Sciences, University of Leicester, Leicester, UK; ${ }^{2}$ Centre for Mathematics Education, Loughborough University, Loughborough, UK; ${ }^{3}$ School of Psychology, Faculty of Science, University of Nottingham, Nottingham, UK; ${ }^{4}$ Institute for Women's Health, University College London, London, UK; ${ }^{5}$ Department of Psychology and Division of Mental Health and Wellbeing, Warwick Medical School, University of Warwick, Coventry, UK. Correspondence: Samantha Johnson (sjj19@le.ac.uk) 
complete the Wechsler Individual Achievement Test-reading (WIAT-RS), whereas two did not complete The Developmental Neuropsychology Test (NEPSY-II) attention/executive functioning subtask and one did not complete the MET. Table 1 shows descriptive statistics for all standardized and experimental tasks.

As expected, EP children underperformed on all measures in comparison to term-born children. There were large effect sizes for between-group differences in accuracy on all measures, except for three of the four MET subcomponents (length, dots, and distance) in which small to medium effect sizes were observed. Not only were control children significantly more accurate on all subcomponents of the MET, they also made significantly fewer erroneous responses to the dot and number line questions (Table 1). Group differences in the magnitude of error for the number line and dots subcomponent also showed large effect sizes. There were no significant sex differences in the control group on any measure. Sex differences were observed for the EP group for mental processing composite (MPC) scores (male: mean $=85.4, \mathrm{SD}=12.2$, female: mean $=89.3, \mathrm{SD}$ $=13.6 ; \mathrm{t}(196)=-2.1, P=0.04)$, Kaufman Assessment Battery for Children $(\mathrm{K}-\mathrm{ABC})$ simultaneous (male: mean $=84.8, \mathrm{SD}=$ 12.5; female: mean $=88.8, \mathrm{SD}=13.6 ; \mathrm{t}(196)=-2.1, P=0.03)$, NEPSY-II sensorimotor (male: mean $=81.7, \mathrm{SD}=15.1$; female: mean $=86.5, \mathrm{SD}=13.1 ; \mathrm{t}(193)=-2.4, P=0.02)$; and NEPSY-II attention/executive function (male: mean $=80.2, \mathrm{SD}=17.2$; female: mean $=90.4, \mathrm{SD}=18.4 ; \mathrm{t}(195)=-3.9, P<0.001)$, with females having higher scores than males. There were no significant differences in any test score between EP children born at $22-24 \mathrm{wk}$ vs. $25 \mathrm{wk}$.

\section{Associations Between Mathematics Attainment and Domain- Specific and Domain-General Measures}

Bivariate correlations between all measures for control and EP children are shown in Table 2. There were significant correlations between the Wechsler Individual Achievement Test-mathematics (WIAT-MS) and all domain-general and domain-specific measures for both control and EP children. However, for the MET subcomponent scores, for control children, the only significant correlation was between WIAT-MS and number line scores. In contrast, for EP children, significant correlations were observed between

Table 1. Descriptive statistics for term-born controls and EP children on standardized and experimental tests

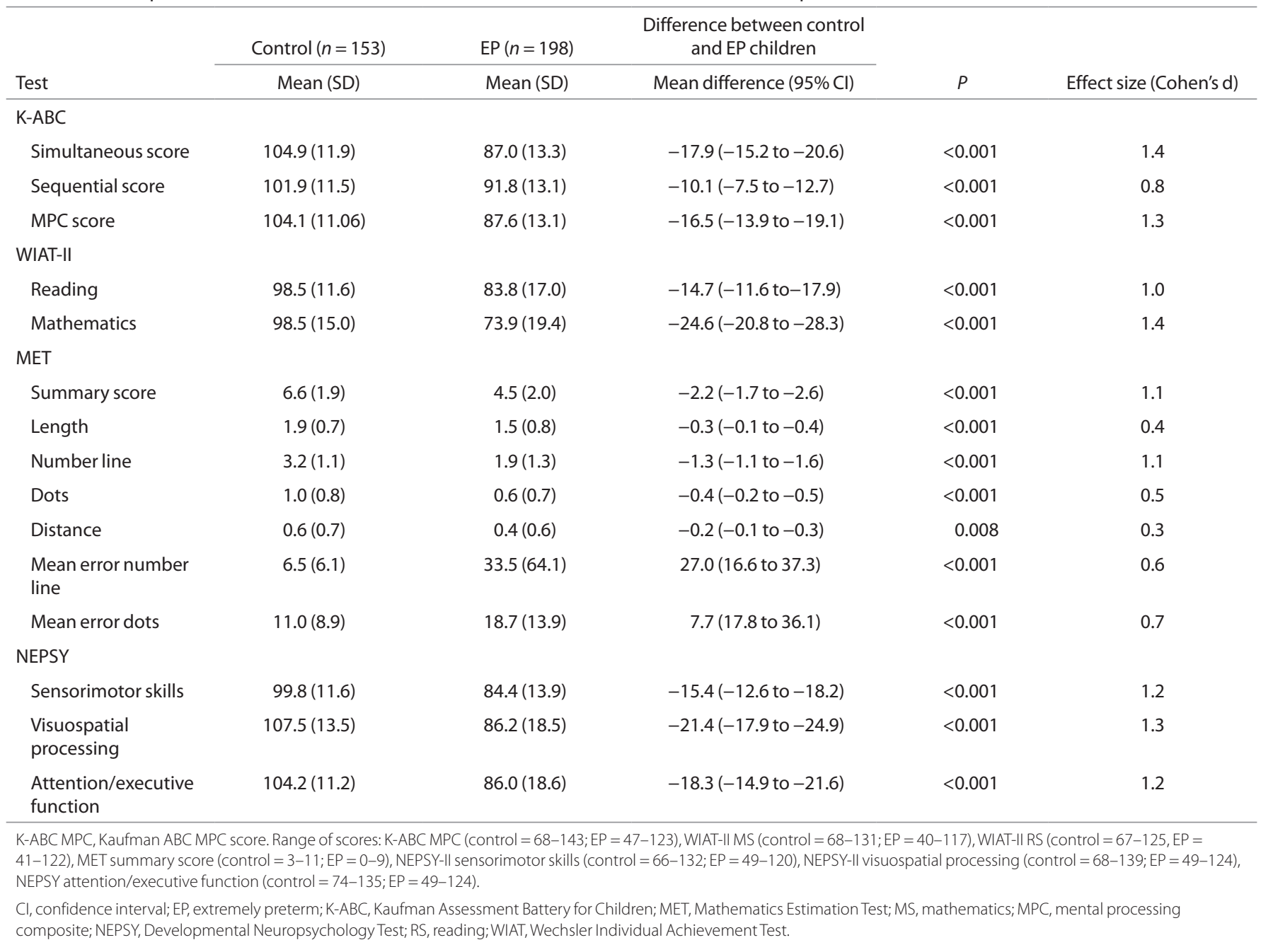




\section{Articles | Simmset al.}

WIAT-MS and number line, dot, and length subcomponents. Fisher $r$-to- $z$ transformations revealed that all correlations between WIAT-MS scores and other measures were significantly stronger for EP than for control children ( $z$ range: -2.61 to -4.75 , all $P<0.05$ ). The most substantial differences in correlations between EP and control children were for WIAT-MS and MET scores $(z=-4.75, P=0.003)$ and WIAT-MS and number line subcomponent scores $(z=$ $-4.58, P<0.001$ ), with significantly stronger associations found for EP children.

Partial correlations were conducted to control for MPC (Table 2). For control children, the only correlations with WIAT-MS scores that remained significant were with WIAT-RS and NEPSY-II visuospatial scores. For EP children, correlations between WIAT-MS scores and the other main measures remained significant $(P<0.05)$. For the domain-specific skills, the correlation with WIAT-MS and both MET total score and number line MET subcomponent remained significant. After adjustment for MPC, the correlation between MET and WIAT-MS was no longer significant for control children $(P$ $=0.250)$, but this correlation remained for EP children $(r=$ $0.346, n=186, P<0.001$ ) (Figure 1).

\section{Predicting Attainment in Mathematics}

Hierarchical stepwise multivariable linear regression was conducted to evaluate the contribution of domain-general (K-ABC simultaneous, K-ABC sequential, WIAT-RS, NEPSY sensorimotor, NEPSY-II visuospatial, and NEPSY-II attention and executive function) and domain-specific (MET) measures to attainment in mathematics (WIAT-MS). This analysis was completed separately for control and $\mathrm{EP}$ children to establish differences in the strength of the contribution of the measures within each group (Table 3). Regression analysis indicated that K-ABC simultaneous, K-ABC sequential, WIAT-RS, and NEPSY visuospatial scores significantly contributed to both EP and control children's attainment in mathematics, explaining a substantial amount of the variance in WIAT-MS scores (control $=48 \%, \mathrm{EP}=72 \%$ ). Simultaneous processing was a stronger predictor of WIAT-MS than sequential processing for EP children; the reverse was true for control children (EP simultaneous: $B=0.30, P=0.001,95 \%$ confidence interval $(\mathrm{CI})=0.12$ to 0.48 ; EP sequential: $B=0.19$, $P=0.02,95 \% \mathrm{CI}=0.03$ to 0.35 ; control simultaneous: $B=0.20$, $P=0.02,95 \% \mathrm{CI}=0.03$ to 0.38 ; control sequential: $B=0.24$, $P=0.01,95 \% \mathrm{CI}=0.05$ to 0.43$)$. MET scores contributed significantly only to EP children's WIAT-MS, explaining an extra $2 \%$ of the variance for this group of children (EP MET: $B=1.59$, $P=0.001,95 \% \mathrm{CI}=0.66$ to 2.52 ; control MET: $B=0.29, P>0.05$, $95 \% \mathrm{CI}=-0.69$ to 1.27 ).

\section{DISCUSSION}

The results of this study confirm those of previous investigations and demonstrate that, by the end of primary education, EP children have markedly poorer attainment in mathematics as compared with children born at term. As expected, termborn control children outperformed EP children on all measures, with large effect sizes for the majority of comparisons.
The observed between-group discrepancies in performance are consistent with previous studies that have reported significant deficits in academic performance in EP children, with the most substantial differences in standardized measures of attainment in mathematics as compared with other school subjects $(3,14,17)$.

This study also confirmed previous research that showed that both literacy skills and visuospatial skills are important for success in mathematics in both EP and control groups as quantified in Table 3 (22-25). The relationship between these domain-general skills and mathematics attainment observed for both groups emphasizes the importance of a wide set of neuropsychological skills in the development of mathematical ability. In contrast, we did not observe a significant contribution to WIAT-MS scores from sensorimotor or attention/ executive skills for either the control or the EP group. This may be surprising in light of previous studies that have suggested the importance of attention, executive functions, and motor skills for success in mathematics (22-24) and academic performance in general (26).

A contrasting relationship between scores on the K-ABC sequential and simultaneous scales was noted for EP and control children. EP children did not perform as well as control children on either of these scales; EP children had greater difficulty with processing simultaneously presented information rather than sequentially presented information, a result replicating those of previous studies $(13,14)$. In fact, simultaneous, rather than sequential, processing score was a stronger predictor of WIAT-MS for the EP group; the reverse was true for control children. Therefore, our results suggest that EP children may have a specific difficulty in integrating information, a skill that appears to be important for mathematical processing.

To our knowledge, this is the first study to investigate specific components of mathematic processing in EP children. Initially, we observed significant correlations with WIAT-MS and MET scores for both control and EP children, indicating a relationship between attainment in mathematics and children's accuracy of numerical representations. This was expected and is consistent with previous studies of typically developing children that demonstrated a relationship between numerical representations and mathematical ability (27).

However, we observed a different relationship between numerical representations and attainment in mathematics for EP and control children. Associations between the measures of attainment and numerical representations were significantly stronger for EP children. After controlling for overall cognitive ability (MPC), the relationship between WIAT-MS and MET scores remained significant for EP children only. This suggests that, in contrast to control children, EP children's attainment in mathematics was associated with their underlying accuracy of numerical representations and was not simply a component of their general cognitive ability. This was further exemplified in the results of the stepwise regression analyses in which MET scores contributed significantly to WIAT-MS scores above the other domain-general measures only for EP children. This study therefore pinpoints that EP children have specific difficulty in 
Table 2. First-order and partial correlations (after controlling for MPC score) between WIAT-II mathematics and other domain-general and domainspecific measures variables for control and EP groups

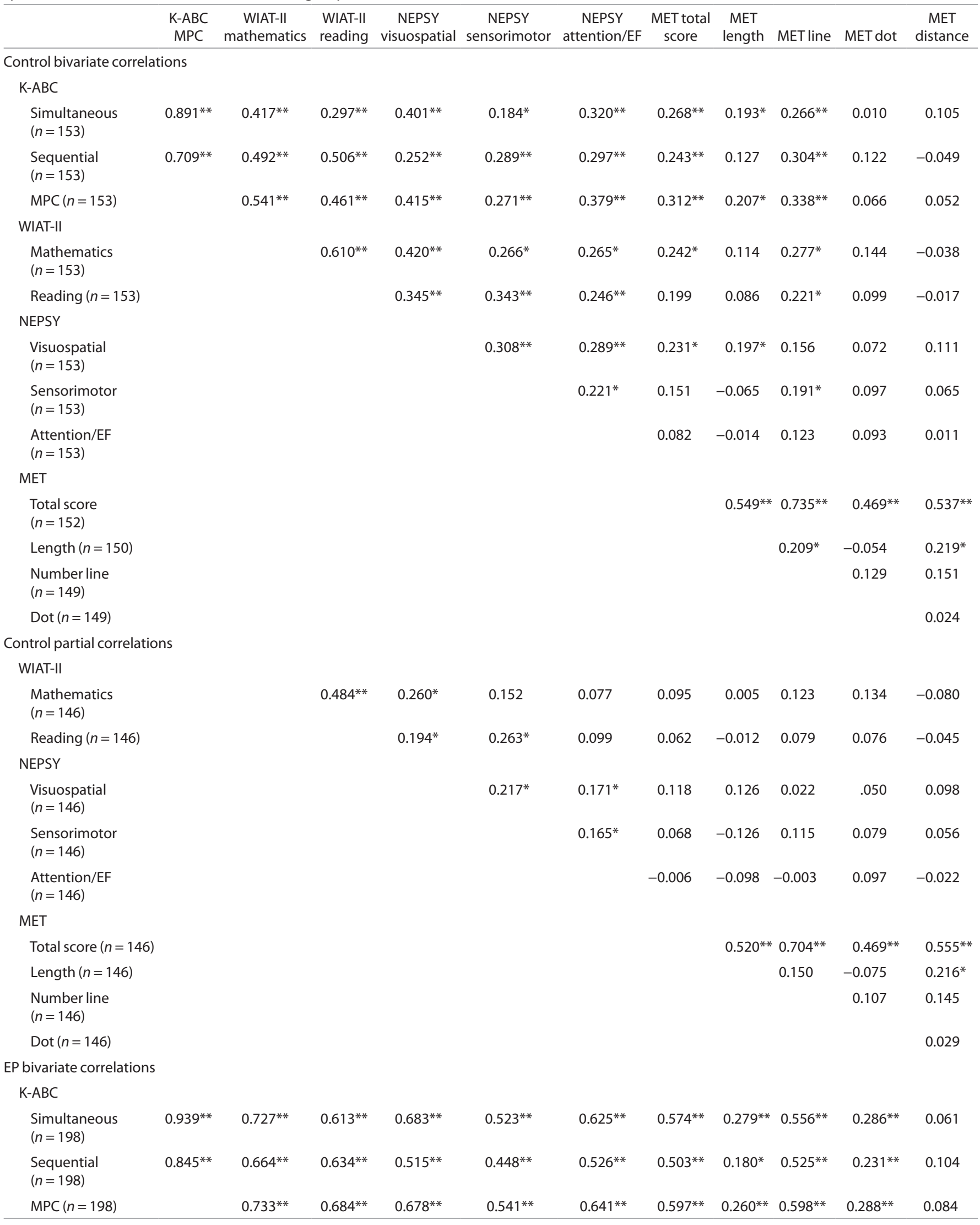




\section{Articles $\mid$ Simmset al.}

Table 2. Continued

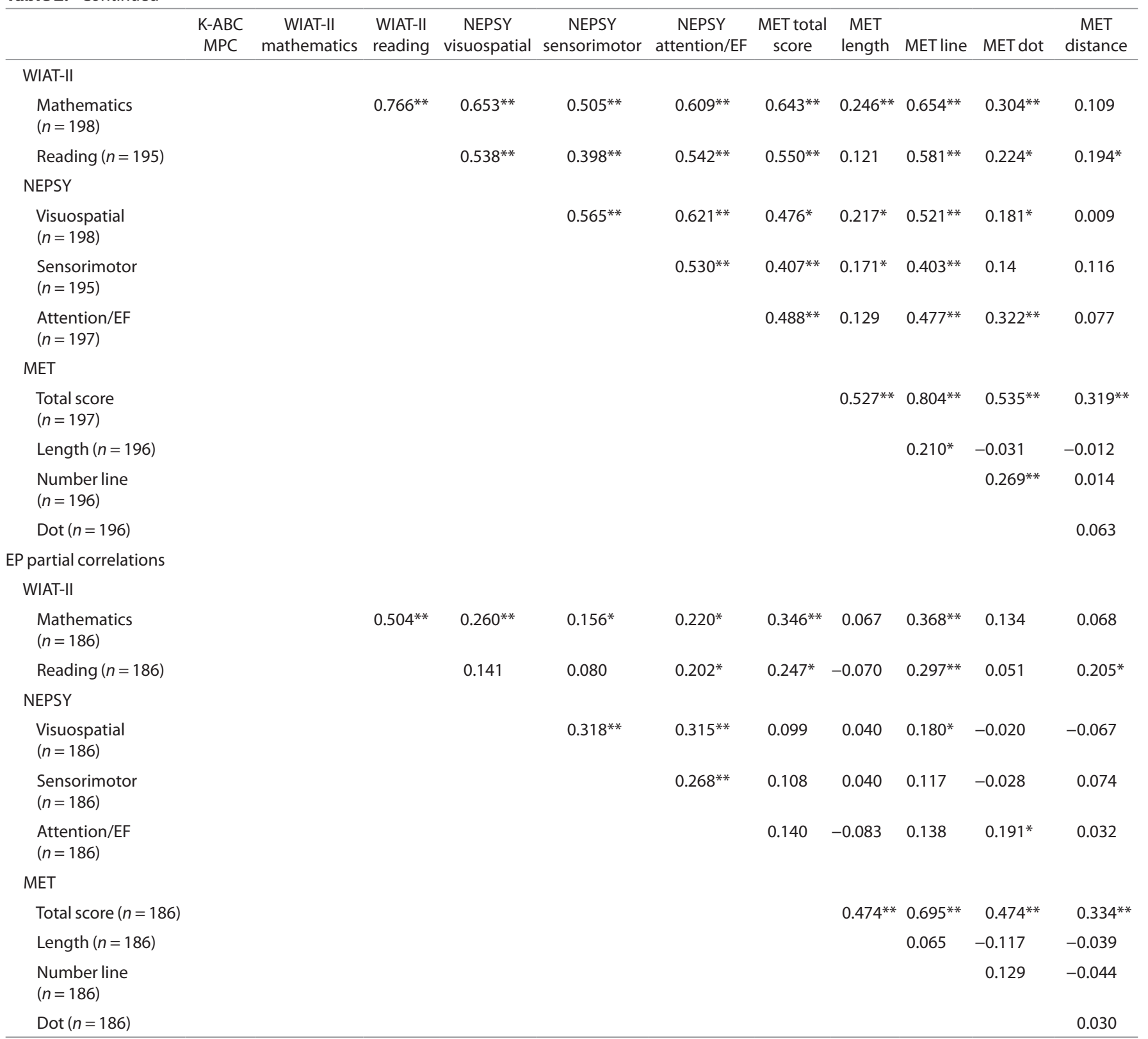

EF, executive functioning; EP, extremely preterm; K-ABC, Kaufman Assessment Battery for Children; MPC, mental processing composite; MET, Mathematics Estimation Test; NEPSY, Developmental Neuropsychology Test;WIAT, Wechsler Individual Achievement Test.

$* P<0.05, * * 0<0.001$.

numerical estimations, a basic mathematical skill, that contributed significantly to their overall mathematical performance. Thus, we have demonstrated that learning difficulties related to mathematics in the EP population may not arise solely as part of the spectrum of domain-general cognitive impairments typically associated with preterm birth, but may involve additional deficits in specific components of mathematical processing that contribute significantly to their underachievement in this area.

Solving mathematical tasks involves different brain areas and the collaboration of large neural networks. The 4CAPS model of complex cognitive neuroarchitecture proposes that when resource demands exceed the resource supply of the first center, processing spills over to less-specialized centers that are now being recruited into the large-scale network (28). Considering that EP children have greater general domain limitations, this spillover is likely to occur sooner and requires the recruitment of specific skills, such as numerical representations. Thus, it appears consistent with the 4CAPS model, that recruiting more centers leads to costs such as bandwidth limitations and more coordination, all of which can be costly for overall performance.

Our finding of the importance of numerical representations for achievement in mathematics in the EP population may perhaps have been expected. A previous neuroimaging study suggested that preterm children's poor magnitude representations may contribute to their overall difficulty in mathematics (29). In addition, it is interesting to note that mathematical difficulties 
a

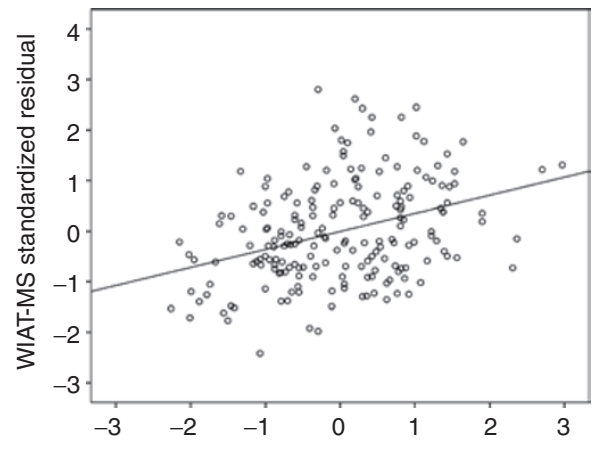

b

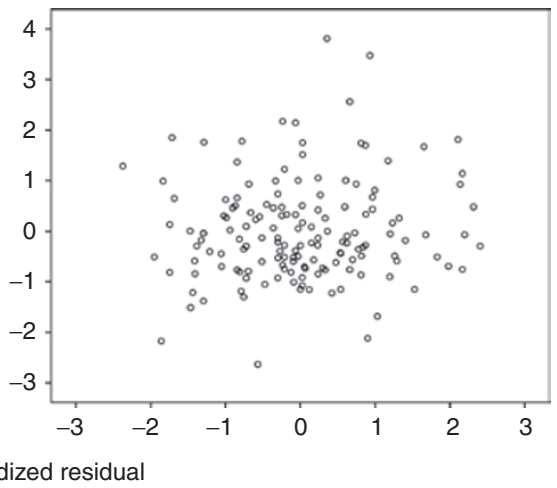

Figure 1. The relationship between WIAT-MS and total MET score standardized residuals for (a) control group and (b) extremely preterm group (regression line: $R^{2}=0.127$ ). MET, Mathematics Estimation Test; WIAT-MS, Wechsler Individual Achievement Test-mathematics.

Table 3. Summary for hierarchical regressions predicting WIAT-MS for control group and EP group

\begin{tabular}{|c|c|c|c|c|c|c|c|}
\hline \multirow[b]{2}{*}{ Model } & \multirow[b]{2}{*}{ Predictor(s) } & \multicolumn{3}{|c|}{ Control } & \multicolumn{3}{|c|}{ EP } \\
\hline & & $R^{2}$ & $\Delta R^{2}$ & B & $R^{2}$ & $\Delta R^{2}$ & B \\
\hline 1 & $\mathrm{~K}-\mathrm{ABC}$ simultaneous & $0.315^{* *}$ & & $0.367^{* *}$ & $0.578^{* *}$ & & $0.728^{* *}$ \\
\hline \multirow[t]{2}{*}{2} & $\mathrm{~K}-\mathrm{ABC}$ simultaneous & $0.458^{* *}$ & $0.143^{* *}$ & $0.279^{*}$ & $0.683^{* *}$ & $0.105^{* *}$ & $0.505^{* *}$ \\
\hline & $\mathrm{K}-\mathrm{ABC}$ sequential & & & $0.256^{*}$ & & & $0.250^{*}$ \\
\hline \multirow[t]{5}{*}{3} & $\mathrm{~K}-\mathrm{ABC}$ simultaneous & $0.479 * *$ & 0.021 & $0.212^{*}$ & $0.700^{* *}$ & $0.017^{*}$ & $0.357^{* *}$ \\
\hline & $\mathrm{K}-\mathrm{ABC}$ sequential & & & $0.250^{*}$ & & & $0.216^{*}$ \\
\hline & WIAT-RS & & & $0.526^{* *}$ & & & $0.463^{* *}$ \\
\hline & NEPSY-II attention/executive & & & 0.012 & & & 0.050 \\
\hline & NEPSY-II sensorimotor & & & -0.018 & & & 0.053 \\
\hline \multirow{5}{*}{4} & WIAT-RS & & & $0.524^{* *}$ & & & $0.415^{* *}$ \\
\hline & NEPSY-II attention/executive & & & 0.016 & & & 0.036 \\
\hline & NEPSY-II sensorimotor & & & -0.020 & & & 0.038 \\
\hline & NEPSY-II visuospatial & & & $0.178^{*}$ & & & $0.137^{*}$ \\
\hline & Total MET score & & & 0.292 & & & $1.589^{*}$ \\
\hline
\end{tabular}

EP, extremely preterm; K-ABC, Kaufman Assessment Battery for Children; MET, Mathematics Estimation Test; NEPSY, Developmental Neuropsychology Test; RS, reading; WIAT, Wechsler Individual Achievement Test.

${ }^{*} P<0.05,{ }^{* *} P<0.001$.

have been associated with poor internal representations of number in other populations of children with neurodevelopmental disorders, for example in children with William's syndrome (30), Down's syndrome (30), and Velio Cardial Facial syndrome (31).

These results suggest that potential educational interventions aiming to improve mathematics attainment in EP children might be best targeted specifically to this population and may involve attempting to improve numerical representations. Indeed, interventions designed to increase children's accuracy of numerical representations have been shown to concurrently improve general mathematical performance (32). However, given the significant association between attainment and other domain-general measures observed in this study, the potential of targeting improvement in these other skills, such as visuospatial skills that require simultaneous information processing, for improving outcomes in mathematics should also be considered. Perhaps EP children would benefit from a combined intervention focusing on both numerical representations and visuospatial skills, in contrast to interventions used with termborn children experiencing pure mathematical difficulties.

The strengths of this study may be attributed to its use of gold-standard contemporary measures of children's cognitive ability and academic attainment, the high level of interrater reliability achieved, and the care taken to ensure psychologists 
Table 4. Description and examples of Mathematics Estimation Test items (37)

\begin{tabular}{|c|c|c|c|}
\hline $\begin{array}{l}\text { Estimation } \\
\text { subcomponent }\end{array}$ & $\begin{array}{l}\text { Number of } \\
\text { questions }\end{array}$ & Example question & Response \\
\hline Length & 3 & $\begin{array}{l}\text { Children were shown an image of three horizontal lines of different lengths } \\
\text { Children were asked: "Here are three lines. Which line is } 5 \mathrm{~cm} \text { long?" }\end{array}$ & $\begin{array}{l}\text { Children were required to point to } \\
\text { the correct line }\end{array}$ \\
\hline Number line & 5 & $\begin{array}{l}\text { Children were shown a blank number line with the start and end number } \\
\text { indicated and an X located on the line } \\
\text { Children were asked: "Here is '0' and here is' } 10 \text {.'Where do you think X is?" }\end{array}$ & $\begin{array}{l}\text { Children were required to state the } \\
\text { value of position } X\end{array}$ \\
\hline Dots & 2 & $\begin{array}{l}\text { Children were shown a set of dots on a single page that varied in quantity } \\
\text { Children were asked:"Look at these spots! How many spots are on this page? Do } \\
\text { you think there are } 20,40,60 \text {, or } 80 \text { spots?" }\end{array}$ & $\begin{array}{l}\text { Children were required to orally } \\
\text { provide the correct quantity }\end{array}$ \\
\hline Distance & 2 & $\begin{array}{l}\text { Children were shown a simple line-drawn map that included a treasure chest, } \\
\text { other locations of interest, and a } 0.5 \mathrm{~cm} \text { line at the top of the page } \\
\text { Children were asked: "If every meter on this map is as long as this (points to the } \\
0.5 \mathrm{~cm} \text { bar), how many meters are there between the tree and the treasure?" }\end{array}$ & $\begin{array}{l}\text { Children were required to orally } \\
\text { provide their approximation of the } \\
\text { distance }\end{array}$ \\
\hline
\end{tabular}

were blinded to the child's birth status. The EP children comprised a large, whole population-based sample drawn from children across the whole of the UK and Ireland who were assessed with a contemporaneous comparator group that achieved a distribution of scores on standardized tests that would be expected of the general population. This is the first time that numerical representations in relation to mathematics abilities has been reported in EP children; however, the MET itself, although sensitive for detecting group differences, is a brief measure. On the other hand, it makes the MET highly usable in both research and school settings. Of course, numerical representations are a single component of a range of separable mathematical processes shown to underlie performance in curriculum-based tests. Thus, future studies should assess a wider range of processes and skills to further investigate the specific difficulties that EP children have with mathematics and the underlying processes associated with these problems.

The results of this study advance our understanding of the likely causes of EP children's difficulties in mathematics and have indicated that one contributing factor may be erroneous numerical representations. A further, more in-depth investigation of preterm children's understanding of mathematics would enable a clearer understanding of why these difficulties occur and what strategies may be effective in improving academic outcomes for these children.

\section{METHODS}

\section{Participants}

Children were recruited from the EPICure Study, a national study of outcome following EP birth (http://www.epicure.ac.uk). All infants born $<26 \mathrm{wk}$ of gestational age in the whole of the UK and Ireland from March through December 1995 and who were admitted for neonatal intensive care $(n=811)$ were invited to participate in the EPICure study. Of surviving children at each time point, $283(90 \%)$ were assessed at $2.5 \mathrm{y}(33), 241(78 \%)$ at $6 \mathrm{y}(34)$, and $219(71 \%)$ at 11 $y$ of age (2).

Analyses for this report used data obtained from follow-up assessments at $11 \mathrm{y}$ of age. At this age, $219 \mathrm{EP}$ children were assessed with a comparison group of 153 children born at term and matched for age, sex, and ethnic group, where possible, to an EP child in mainstream school. Controls were not selected for children in special schools.
A detailed description of the full sample at $11 \mathrm{y}$ was published previously (3).Of these 219, $21 \mathrm{EP}$ children were unable to complete the full battery of mathematics tests and were excluded from this study. Reasons for exclusion were as follows: functioning below the level of the test (14 children), blindness ( 2 children), attention difficulties (2 children), autism (1 child), limited language (1 child), and poor motor skills ( 1 child). Of the excluded children, 19 were classified as having a serious disability. Participants thus comprised 198 EP children (mean age at assessment $131.1 \mathrm{mo}$; SD $4.5 \mathrm{mo}$; range: $121-145 \mathrm{mo}$; males: 43\%) and 153 term-born control children (mean age at assessment 131.2 mo; SD: 6.6 mo; range: $117-147$ mo; males: $42 \%$ ). There were no significant differences in mean age at assessment $(\mathrm{t}(349)=0.3, P$ $=0.781)$ or sex $(\mathrm{t}(351)=0.1, P=0.8)$ between $\mathrm{EP}$ children and controls. Of EP children, $34.8 \%$ had a cognitive impairment (intelligence quotient) score $<-2 \mathrm{SD}$ of control reference data measured with the K-ABC MPC (35), and 4.5\%, 5.6\%, and $1 \%$ had a motor, vision, or hearing impairment, respectively. In contrast, $1.3 \%$ of term-controls had a cognitive impairment and none had visual, hearing, or motor impairments.

\section{Procedure}

Parents and children received study information leaflets and parents provided informed consent for their child's participation at $11 \mathrm{y}$ of age. Children were assessed individually by a psychologist in a quiet area in the child's school (92\%), at their home (7\%), or a hospital (1\%). Psychologists had no prior knowledge of the child and were blinded to study group allocation. The study was approved by the Southampton and South West Hampshire research ethics committee.

\section{Measures}

One of three study psychologists administered the reading and mathematics scales of the WIAT-II UK (36). This is the most contemporary standardized test of curriculum-based attainment from which standardized scores (mean 100; SD 15; range 40-160) were derived for attainment in reading and mathematics. WIAT-RS subscales assessed reading comprehension, word reading, and pseudo-word decoding. WIAT-MS subscales comprised numerical operations (paper and pencil test of performance in simple operations such as addition or subtraction) and mathematical reasoning (orally presented test of ability to apply mathematics in everyday scenarios, e.g., telling the time, using money).

To assess domain-specific numerical representations, children completed the English version of the MET (37) previously used with very preterm and full-term children in a German sample. This task was presented to children in book form and required oral or manual responses to 12 items assessing approximations of four subcomponents of numerical estimations: length, number line, dot, and distance (Table 4). Item responses were scored for accuracy and a total score (range 0-12) was summed in addition to summary scores for each of the four subcomponents of the test. Error scores for the number line and dot tasks were also 
calculated by subtracting the correct answer from the child's response in order to establish the magnitude of error on these tasks.

Two tests of domain-general abilities were administered. Intelligence quotient was assessed using the K-ABC (35). The K-ABC comprises eight age-appropriate subtests that generate two separate global scales: sequential (three subtests) and simultaneous (five subtests) processing. These two global scales were also combined into a MPC (standardized mean 100; SD 15; range 40-160) score for global cognitive ability (intelligence quotient). Children also completed the NEPSY-II (38), a standardized developmental neuropsychological test battery. Standardized scores (mean 100; SD 15; range 50-150) for sensorimotor, visuospatial processing, and attention and executive functioning were derived. Psychologists achieved excellent interrater reliability on all tests (agreement on $>95 \%$ item scores) before commencing data collection.

\section{Statistical Analyses}

Data were double entered, verified, and analyzed using SPSS v18.0 (Chicago, IL). Independent-samples' $t$-tests were used to compare performance on all measures between EP and control children and Cohen's d was calculated to determine standardized effect sizes across tests. Effect sizes were defined as small (0.2-0.3), medium (0.3-0.5), or large $(>0.5)$ (39). Bivariate correlations (two-tailed) between all measures were conducted for EP and control children separately, and partial correlations (two-tailed) were conducted controlling for MPC. Fischer $r$-to- $z$ transformations were also calculated to assess the difference in magnitude between correlations for the EP and control group on the same measures. Separate hierarchical stepwise multivariable linear regressions were conducted for control and EP children to identify predictors of mathematics attainment. WIAT-MS was the dependent variable, and independent variables were entered in the following order (domain general to domain specific): Step 1, $\mathrm{K}-\mathrm{ABC}$ simultaneous and sequential processing; Step 2 WIAT-RS; Step 3 NEPSY-II sensorimotor, visuospatial processing, and attention and executive functioning; Step 4 MET.

\section{ACKNOWLEDGMENT}

Summary data used in this paper have been published previously $(3,9,14)$, but the analysis presented here takes a different approach to the data in order to more fully understand the specific difficulty that very preterm children have with mathematics, and to tease out factors that may contribute to the observed problems in mathematical processing for this group.

\section{STATEMENT OF FINANCIAL SUPPORT}

This study was funded by the Medical Research Council, London, UK.

Disclosure: The authors declare no conflict of interest.

\section{REFERENCES}

1. Field DJ, Dorling JS, Manktelow BN, Draper ES. Survival of extremely premature babies in a geographically defined population: prospective cohort study of 1994-9 compared with 2000-5. BMJ 2008;336:1221-3.

2. Johnson S, Fawke J, Hennessy E, et al. Neuro-developmental disability through 11 years of age in children born before 26 weeks of gestation. Pediatrics 2009; 124:e249-57.

3. Johnson S, Hennessy E, Smith R, Trikic R, Wolke D, Marlow N. Academic attainment and special educational needs in extremely preterm children at 11 years of age: the EPICure study. Arch Dis Child Fetal Neonatal Ed 2009;94:F283-9.

4. Anderson PJ, De Luca CR, Hutchinson E, Spencer-Smith MM, Roberts G, Doyle LW; Victorian Infant Collaborative Study Group. Attention problems in a representative sample of extremely preterm/extremely low birth weight children. Dev Neuropsychol 2011;36:57-73.

5. Mulder H, Pitchford NJ, Hagger MS, Marlow N. Development of executive function and attention in preterm children: a systematic review. Dev Neuropsychol 2009;34:393-421.

6. Marlow N, Hennessy EM, Bracewell MA, Wolke D. Motor and executive function at 6 years of age after extremely preterm birth. Pediatrics 2007;120:793-804.
7. Luciana M, Lindeke L, Georgieff M, Mills M, Nelson CA. Neurobehavioral evidence for working-memory deficits in school-aged children with histories of prematurity. Dev Med Child Neurol 1999;41:521-33.

8. Rose SA, Feldman JF. Memory and processing speed in preterm children at eleven years: a comparison with full-terms. Child Dev 1996;67:2005-21.

9. Johnson S, Wolke D, Hennessy E, Marlow N. Educational outcomes in extremely preterm children: neuropsychological correlates and predictors of attainment. Dev Neuropsychol 2011;36:74-95.

10. Taylor HG, Klein N, Anselmo MG, Minich N, Espy KA, Hack M. Learning problems in kindergarten students with extremely preterm birth. Arch Pediatr Adolesc Med 2011;165:819-25.

11. Woodward LJ, Moor S, Hood KM, et al. Very preterm children show impairments across multiple neurodevelopmental domains by age 4 years. Arch Dis Child Fetal Neonatal Ed 2009;94:F339-44.

12. Hornby G, Woodward LJ. Educational needs of school-aged children born very and extremely preterm: a review. Educ Psychol Rev 2009; 21:247-66.

13. Wolke D, Meyer R. Cognitive status, language attainment, and prereading skills of 6-year-old very preterm children and their peers: the Bavarian Longitudinal Study. Dev Med Child Neurol 1999;41:94-109.

14. Johnson S, Marlow N, Wolke D. Assessing educational outcomes in middle childhood: validation of the Teacher Academic Attainment Scale. Dev Med Child Neurol 2012;54:544-51.

15. Taylor G, Espy KA, Anderson PJ. Mathematics deficiencies in children with very Low Birth Weight or Very Preterm Birth. Dev Disabil Res Rev 2009;15:52-9.

16. Anderson P, Doyle LW; Victorian Infant Collaborative Study Group. Neurobehavioral outcomes of school-age children born extremely low birth weight or very preterm in the 1990s. JAMA 2003;289:3264-72.

17. Wocadlo C, Rieger I. Phonology, rapid naming and academic achievement in very preterm children at eight years of age. Early Hum Dev 2007;83:367-77.

18. Geary DC, Hoard MK, Byrd-Craven J, Nugent L, Numtee C. Cognitive mechanisms underlying achievement deficits in children with mathematical learning disability. Child Dev 2007;78:1343-59.

19. Mazzocco MM, Singh Bhatia N, Lesniak-Karpiak K. Visuospatial skills and their association with math performance in girls with fragile $\mathrm{X}$ or Turner syndrome. Child Neuropsychol 2006;12:87-110.

20. Bull R, Espy KA, Wiebe SA. Short-term memory, working memory, and executive functioning in preschoolers: longitudinal predictors of mathematical achievement at age 7 years. Dev Neuropsychol 2008;33:205-28.

21. LeFevre JA, Fast L, Skwarchuk SL, et al. Pathways to mathematics: longitudinal predictors of performance. Child Dev 2010;81:1753-67.

22. Geary DC. Mathematical disabilities: cognitive, neuropsychological, and genetic components. Psychol Bull 1993;114:345-62.

23. Torbeyns J, Verschaffel L, Ghesquière P. Strategy development in children with mathematical disabilities: insights from the choice/no-choice method and the chronological-age/ ability-level-match design. J Learn Disabil 2004;37:119-31.

24. Geary DC, Brown SC. Cognitive addition: Strategy choice and speedof-processing differences in gifted, normal, and mathematically disabled children. Dev Psychol 1991;27:398-406.

25. De Smedt B, Verschaffel L, Ghesquière P. The predictive value of numerical magnitude comparison for individual differences in mathematics achievement. J Exp Child Psychol 2009;103:469-79.

26. Jaekel J, Wolke D, Bartmann P. Poor attention rather than hyperactivity/impulsivity predicts academic achievement in very preterm and full-term adolescents. Psychol Med 2012; e-pub ahead of print 21 May 2012.

27. Gilmore CK, McCarthy SE, Spelke ES. Non-symbolic arithmetic abilities and mathematics achievement in the first year of formal schooling. Cognition 2010;115:394-406.

28. Just MA, Varma S. The organization of thinking: what functional brain imaging reveals about the neuroarchitecture of complex cognition. Cogn Affect Behav Neurosci 2007;7:153-91.

29. Isaacs EB, Edmonds CJ, Lucas A, Gadian DG. Calculation difficulties in children of very low birthweight: a neural correlate. Brain 2001; 124(Pt 9):1701-7. 


\section{Articles $\mid$ Simmset al.}

30. Paterson SJ, Girelli L, Butterworth B, Karmiloff-Smith A. Are numerical impairments syndrome specific? Evidence from Williams syndrome and Down's syndrome. J Child Psychol Psychiatry 2006; 47:190-204.

31. De Smedt B, Swillen A, Devriendt K, Fryns JP, Verschaffel L, Ghesquière P. Mathematical disabilities in children with velo-cardio-facial syndrome. Neuropsychologia 2007;45:885-95.

32. Ramani GB, Siegler RS. Reducing the gap in numerical knowledge between low- and middle-income preschoolers. J Applied Dev Psychol 2011;32:146-159.

33. Wood NS, Costeloe K, Gibson AT, Hennessy EM, Marlow N, Wilkinson AR. The EPICure study: associations and antecedents of neurological and developmental disability at 30 months of age following extremely preterm birth. Arch Dis Child Fetal Neonatal Ed 2005;90:F134-40.
34. Marlow N, Wolke D, Bracewell M, Samara M. Neurologic and developmental disability at 6 years of age following extremely preterm birth. New Engl J Med 2005;352:9-19.

35. Kaufman, AS, Kaufman, NL. Kaufman-ABC, 2nd edn. Circle Pines, MN: American Guidance Service, 2004.

36. Weschler D. Weschler Individual Achievement Test. 2nd edn. Oxford, UK: Pearson Assessment/PsychCorp, 2005.

37. Wolke D, Schulz J, Meyer R. Entwicklungslangzeitfolgen bei ehemaligen, sehr unreifen Fruehgeborenen [long term developmental outcome of ex very preterm born children]. Monatsschrift fuer Kinderheilkunde 2001;149:S53-61.

38. Korkman M, Kirk U, Kemp S. NEPSY: A developmental neuropsychological assessment. San Antonio, TX: The Psychological Corporation, 1998.

39. Cohen J. Statistical Power Analysis for the Behavioral Sciences. 2nd edn. Hillsdale, NJ: Lawrence Erlbaum Associates, 1988:25. 